\title{
Exploring the Neurodynamic Signals of the Deafened Brain: Factors Influencing Cochlear Implant Outcomes
}

\author{
Anu Sharma ${ }^{1}$ (i) $\cdot$ Jeong-Sug Kyong ${ }^{2,3}$ (i) \\ ${ }^{I}$ Department of Speech Language, and Hearing Science, University of Colorado Boulder, Boulder, CO, USA, ${ }^{2}$ Medical Research Center, Seoul \\ National University College of Medicine, Seoul; ${ }^{3}$ Audiology Institute, Hallym University of Graduate Studies, Seoul, Korea
}

Deafness alters the neurodynamics of central processing within and across sensory modalities, thereby impacting real-world speech communication. Temporal processing (i.e., processing of acoustic objects that vary over time) constitutes an essential element for understanding speech. Distorted or damaged auditory temporal processing impairs speech-related activity, delaying development in children and increasing listening effort in adults with hearing loss. Even in normal-hearing adults, speech occurring in noisy conditions may result in temporal masking and masking of the target signal itself, requiring temporal segregation between the two for comprehension to be possible. This adversity is a consistent challenge in cochlear implant (CI) users, resulting in impaired speech-in-noise ( $\mathrm{SiN}$ ) perception due to the inherent spectral and temporal limitations of CIs.

Since SiN perception is not sufficiently estimated by the individual hearing threshold and varies among CI users, individual central auditory processing is suggested to be more closely associated with variability in SiN perception [1]. Han et al. [2] investigated the relationship between behavioral and electrophysiological measures of SiN perception. Using varied voice onset times, they observed that difficulties in SiN were revealed even in good performers, particularly in words with low familiarity. Moreover, N1/P2 cortical auditory evoked potential latencies increased with noise masking, and the electrophysiological component P2 (rather than N1) was suggested to better reflect SiN in CI users. They concluded that active learners have more benefits from CI, as evidenced by shortened P2 latencies. We may not preclude the possibility that this finding is affected by age, since age differences were not systematically controlled, but the meaningful contribution of this study lies in its objective quantification of cortical auditory activity in response to temporal features, helping gauge the patient's current status and providing guidance for any adjustments or need for alternate interven- tions.

Recently, factors beyond central auditory processing were reported to influence CI outcomes. When auditory input is decreased and/or degraded, auditory cortical areas may be repurposed by other intact modalities, such as the visual and somatosensory modalities. This process, referred to as cross-modal neuroplasticity, may contribute to the outcomes of CIs. In congenitally and profoundly deaf adults, Scott et al. [3] observed reliably increased responses to visual stimulation in Heschl's gyrus regions, the site of the primary auditory cortex, unlike matched hearing adults, using functional magnetic resonance imaging. More recently, earlier and larger cortical visual and somatosensory evoked potentials and the corresponding activation of auditory cortical areas by visual [4] and somatosensory [5] stimuli were observed in pediatric CI users compared to age-matched controls, indicative of cross-modal reorganization. These crossmodal changes were negatively correlated with $\mathrm{SiN}$ perception, in that children who showed more cross-modal plasticity also had more difficulty with SiN, suggesting that cross-modal reorganization is an important factor influencing CI outcomes. This was confirmed by a recent case study demonstrating that a highly plastic auditory cortex and consequent reversal of cross-modal reorganization after CI were associated with good outcomes in a single-sided deaf child, demonstrating the clinical feasibility of these techniques [6].

Using newer imaging methods such as functional near-infrared spectroscopy, Anderson et al. [7] observed increased visual activation in response to sound in poor CI performers, suggesting that the degree of preoperational visual activation may function as a predictive factor of CI outcomes. Thus, while re-organization during deafness appears to limit speech perception outcomes with a $\mathrm{CI}$, a recent study showed a positive relationship between cross-modal activation and audiovisual integration

Copyright (C) 2020 by Korean Society of Otorhinolaryngology-Head and Neck Surgery.

This is an open-access article distributed under the terms of the Creative Commons Attribution Non-Commercial License (https://creativecommons.org/licenses/by-nc/4.0) which permits unrestricted non-commercial use, distribution, and reproduction in any medium, provided the original work is properly cited. 
strength for CI users, suggesting that the associated benefits in face recognition and lip-reading may be useful in real-world communication [8].

Various neurodynamic cues in the deafened brain are being investigated in terms of CI outcome measures, with potential predictive roles and the possibility to serve as a guide to future rehabilitation. Since advances in CI technology have already enabled phonetic-level segregation, future efforts should focus on aural rehabilitation tailored towards understanding and utilizing multi-sensory cortical processing to optimize CI outcomes.

\section{CONFLICT OF INTEREST}

No potential conflict of interest relevant to this article was reported.

\section{ACKNOWLEDGMENTS}

This work was supported by the National Research Foundation of Korea (NRF-2018R1A2B6004788).

\section{ORCID}

Anu Sharma

https://orcid.org/0000-0001-8568-1420

Jeong-Sug Kyong

https://orcid.org/0000-0003-0798-0059
Methodology: all authors. Project administration: JSK. Visualization \& Writing-original draft: all authors. Writing-review \& editing: JSK.

\section{REFERENCES}

1. Bidelman GM, Howell M. Functional changes in inter- and intra-hemispheric cortical processing underlying degraded speech perception. Neuroimage. 2016 Jan;124(PtA):581-90.

2. Han JH, Lee J, Lee HJ. Noise-induced change of cortical temporal processing in cochlear implant users. Clin Exp Otorhinolaryngol. 2020 Aug;13(3):241-8.

3. Scott GD, Karns CM, Dow MW, Stevens C, Neville HJ. Enhanced peripheral visual processing in congenitally deaf humans is supported by multiple brain regions, including primary auditory cortex. Front Hum Neurosci. 2014 Mar;8:177.

4. Campbell J, Sharma A.Visual cross-modal re-organization in children with cochlear implants. PLoS One. 2016 Jan;11(1):e0147793.

5. Cardon G, Sharma A. Somatosensory cross-modal reorganization in children with cochlear implants. Front Neurosci. 2019 Jun;13:469.

6. Sharma A, Glick H, Campbell J, Torres J, Dorman M, Zeitler DM. Cortical plasticity and reorganization in pediatric single-sided deafness pre- and postcochlear implantation: a case study. Otol Neurotol. 2016 Feb;37(2):e26-34.

7. Anderson CA, Wiggins IM, Kitterick PT, Hartley DE. Pre-operative brain imaging using functional near-infrared spectroscopy helps predict cochlear implant outcome in deaf adults. J Assoc Res Otolaryngol. 2019 Oct;20(5):511-28.

8. Stropahl M, Debener S. Auditory cross-modal reorganization in cochlear implant users indicates audio-visual integration. Neuroimage Clin. 2017 Sep;16:514-23.

\section{AUTHOR CONTRIBUTIONS}

Conceptualization: JSK. Data curation: AS. Formal analysis \& 